\title{
Border Ownership Selectivity in Human Early Visual Cortex and its Modulation by Attention
}

\author{
Fang Fang, ${ }^{1}$ Huseyin Boyaci, ${ }^{2}$ and Daniel Kersten ${ }^{3}$ \\ ${ }^{1}$ Department of Psychology and Key Laboratory of Machine Perception (Ministry of Education), Peking University, Beijing 100871, China, ${ }^{2}$ Department of \\ Psychology, Bilkent University, Ankara 06800, Turkey, and ${ }^{3}$ Department of Psychology, University of Minnesota, Minneapolis, Minnesota 55455
}

\begin{abstract}
Natural images are usually cluttered because objects occlude one another. A critical aspect of recognizing these visual objects is to identify the borders between image regions that belong to different objects. However, the neural coding of border ownership in human visual cortex is largely unknown. In this study, we designed two simple but compelling stimuli in which a slight change of contextual information could induce a dramatic change of border ownership. Using functional MRI adaptation, we found that border ownership selectivity in V2 was robust and reliable across subjects, and it was largely dependent on attention. Our study provides the first human evidence that $\mathrm{V} 2$ is a critical area for the processing of border ownership and that this processing depends on the modulation from higher-level cortical areas.
\end{abstract}

Key words: visual cortex; fMRI; vision; figure-ground segregation; attention; border ownership

\section{Introduction}

Border ownership is a term for the phenomenon that a visual border between two image regions is normally perceived to belong to only one of the regions. Border ownership assignment determines the figure-ground organization in a visual image and it is a critical aspect of object recognition (Nakayama et al., 1989; Driver and Baylis, 1996). Primate electrophysiological studies (Zhou et al., 2000; Qiu and von der Heydt, 2005) have shown that neurons in the early visual cortex encode the side to which a border belongs. Human functional imaging studies (Kourtzi and Kanwisher, 2001; Andrews et al., 2002) have demonstrated that higher-level visual areas lateral occipital complex (LOC) and fusiform face area (FFA) are sensitive to a change of border ownership, but to date have provided no evidence regarding border ownership selectivity in human early visual cortex.

We designed our stimuli (see Fig. $1 A$ ) by modifying a bright/ dark square-wave radial grating annulus. In the stimuli, either the bright or dark stripes (sectors of a disk) are slightly longer in the radial direction, both inward and outward. This provides contextual information that causes the borders between the bright and the dark stripes to appear to belong either to the bright stripes or the dark stripes, respectively. Although the image difference (the contextual information) between the two stimuli is very small, it dramatically changes the border ownership of locally identical edges along the edges of the stripes. With these two stimuli, we attempted to address three specific questions: (1) Are neurons in

Received Sept. 26, 2008; revised Dec. 5, 2008; accepted Dec. 8, 2008.

This work was supported by National Institutes of Health Grant EY015261 and the National Natural Science Foundation of China (Project 30870762). The 3T scanner at the University of Minnesota, Center for Magnetic Resonance Research, was supported by National Center for Research Resources P41 008079 and P30 NS057091 and by the MIND Institute.

Correspondence should be addressed to Fang Fang, Department of Psychology and Key Laboratory of Machine Perception (Ministry of Education), Peking University, Beijing 100871, China. E-mail: ffang@pku.edu.cn.

D01:10.1523/JNEUROSCI.4628-08.2009

Copyright $\odot 2009$ Society for Neuroscience $\quad$ 0270-6474/09/290460-06\$15.00/0 human early visual cortical areas selective for border ownership due to contextual modulation? (2) If so, is there any selectivity difference between the striate cortex (V1) and extrastriate cortical areas (e.g., V2)? (3) What is the role of attention in the processing of border ownership?

Since the border ownership selective neurons, if any, are very likely to mix with each other below functional MRI (fMRI) spatial resolution, we used fMRI adaptation to overcome this difficulty. fMRI adaptation has been demonstrated to provide insight into the functional properties of subpopulations of neurons within an imaging voxel (Fang et al., 2005; Krekelberg et al., 2006). To address the third question, we used two distinct attentional tasks to examine how manipulating attention modulates the border ownership selectivity of early cortical areas. Subjects were asked to attend to either the stimulus or a fixation point.

\section{Materials and Methods}

Subjects. Four subjects ( 3 male and 1 female) participated in all the experiments. All of them were right-handed, reported normal or correctedto-normal vision, and had no known neurological or visual disorders. Ages ranged from 26 to 36 . They gave written, informed consent in accordance with procedures and protocols approved by the human subjects review committee of the University of Minnesota.

Stimuli and designs. The two stimuli (Fig. $1 A$ ) used in the main experiment were generated by modifying a square-wave radial grating with 18 cycles per revolution and Michelson contrast of 0.8 . The inner and outer radii of the grating were $1.21^{\circ}$ and $7.46^{\circ}$ respectively. In one stimulus, the bright stripes were slightly elongated in the radial direction both inward and outward by $0.35^{\circ}$. This provided contextual information that caused the borders between the bright and the dark stripes to appear to belong to the bright stripes. In the other stimulus, the dark stripes were elongated in the radial direction both inward and outward by $0.35^{\circ}$, which caused the borders to appear to belong to the dark stripes.

For the stimuli used in the control experiment (Fig. $1 B$ ), the areas between $1.21^{\circ}$ and $3.69^{\circ}$ eccentricity and between $4.71^{\circ}$ and $7.46^{\circ}$ eccentricity were filled with a mean luminance of $120 \mathrm{~cd} / \mathrm{m}^{2}$. The other parts were the same as those in the stimuli used in the main experiment. So the 


\section{A. Stimuli in main experiment}
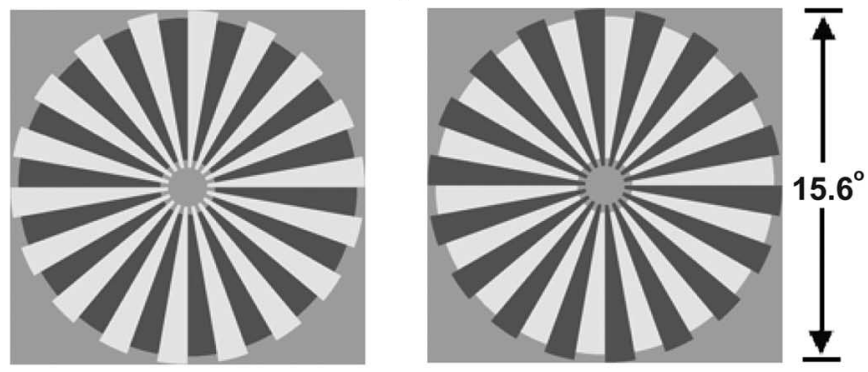

\section{B. Stimuli in control experiment}
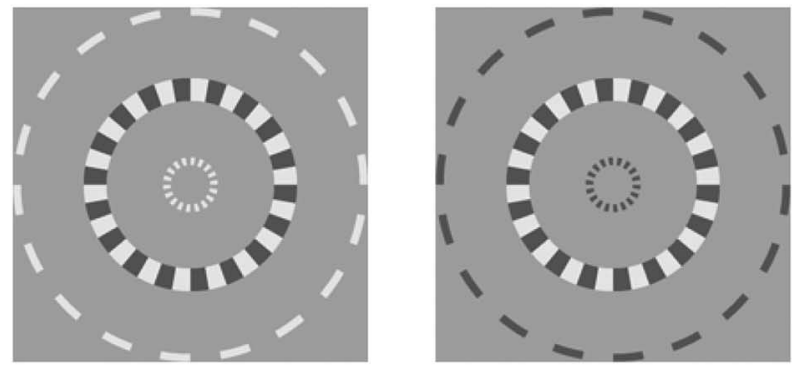

\section{ROI}
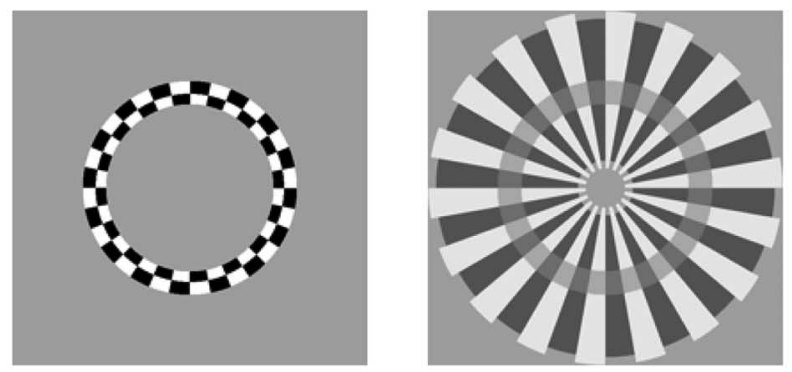

D. Procedure
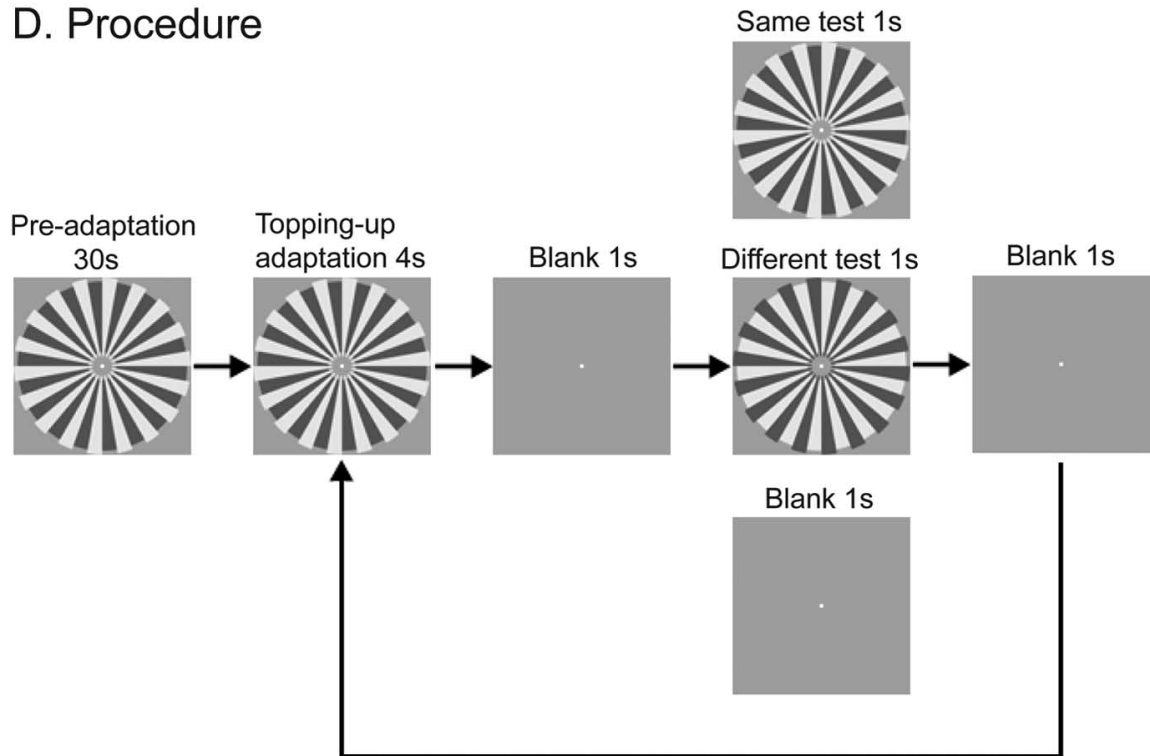

Figure 1. Stimuli and designs. $A$, Stimuli used in the main experiment. The interior part of the stimuli was locally identical across the two stimuli, but as a consequence of the difference in the contextual information, the borders between the bright and the dark stripes were perceived to belong to either the bright or the dark stripes. $\boldsymbol{B}$, Stimuli used in the control experiment. The image difference between the stimulus pair in the control experiment was the same as that in the main experiment, but the borders between the bright and the dark stripes do not have a clear ownership. C, ROI definition. The checkered ring in the left panel was used to define R0Is in V1 and V2. The transparent gray ring in the right panel shows the size of the checkered ring relative to the stimulus in the main experiment. $\boldsymbol{D}$, Schematic description of the experimental procedure. The example here illustrates three trial types: same, different and blank. image difference between the stimulus pair in the control experiment was the same as that in the main experiment. The checkered ring stimulus (Fig. 1C) used to define the regions of interest (ROI) in V1 and V2 had the same size as the rings in the stimuli used in the control experiment, with inner and outer radii of $3.69^{\circ}$ and $4.71^{\circ}$, respectively.

The main experiment consisted of an attendto-stimulus condition and an attend-tofixation condition. Each $410 \mathrm{~s}$ adaptation scan (six in one session for the attend-to-stimulus condition and six in another session for the attend-to-fixation condition) consisted of 54 continuous trials and began with 30 s preadaptation (Fig. 1D). There were three types of trials: same, different and blank trials. In the same and different trials, after a 4 s topping-up adaptation and $1 \mathrm{~s}$ blank interval, a test stimulus was presented for $1 \mathrm{~s}$, followed by a $1 \mathrm{~s}$ blank interval. In the blank trials, a $4 \mathrm{~s}$ topping-up adaptation was followed by $3 \mathrm{~s}$ blank interval. One of the two stimuli in Figure $1 A$ was used as adapting stimulus in three scans and the other was used in the other three scans. Both stimuli were used as test stimuli in all six scans. In the same trials, the adapting stimulus was the same as the test stimulus. In the different trials, the adapting stimulus was different from the test stimulus. Although the stimuli in the attend-tostimulus condition and in the attend-tofixation condition were the same, subjects performed different tasks. In the attend-tostimulus condition, they were asked to passively view the adapting stimulus and to identify the test stimulus as one of the two stimuli as quickly as possible by pressing one of two buttons. In the attend-to-fixation condition, the subjects performed a very demanding fixation task in which they needed to press one of two buttons to indicate the $200 \mathrm{~ms}$ luminance change (increase or decrease) of the fixation point as quickly as possible. The luminance changes occurred randomly and approximately every 1-1.2 s across the whole scan. Subjects reported having little awareness of the peripheral stimulus while performing this task. For the control experiment, the experimental procedure was the same as that in the attend-to-stimulus condition except the stimuli (Fig. $1 B$ ). To avoid retinal adaptation and the formation of afterimage, all the test and adapting stimuli rotated back and forth within a range of $\pm 5^{\circ}$ at a speed of $2.5 \%$ s.

For all of these event-related experiments, there were a total of $18 \times 6$ trials, 108 for each type of trial. The order of the three types of trials (same, different and blank) was counterbalanced across 6 adaptation scans using M-sequences (Buracas and Boynton, 2002). These are pseudo-random sequences which have the advantage of being perfectly counterbalanced $n$ trials back, so that each type of trial was preceded and followed equally often by all types of trials, including itself.

Retinotopic visual areas were defined by a standard phase-encoded method developed by Sereno et al. (1995) and Engel et al. (1997), in which subjects viewed rotating wedge and ex- 
panding ring stimuli that create traveling waves of neural activity in visual cortex. A blockdesign scan was used to define the ROI in V1 and V2. The scan consisted of five $15 \mathrm{~s}$ stimulus blocks interleaved with five $15 \mathrm{~s}$ blank intervals. In a stimulus block, subjects passively viewed a $5 \mathrm{~Hz}$ counterphase-flickering checkered ring (Fig. 1C).

MRI data acquisition. In the scanner, the stimuli were back-projected via a video projector (60 $\mathrm{Hz}$ ) onto a translucent screen placed inside the scanner bore. Subjects viewed the stimuli through a mirror located above their eyes. The viewing distance was $92 \mathrm{~cm}$. MRI data were collected using a 3T Siemens Trio scanner with an eight-channel phase-array coil. Blood oxygen level-dependent (BOLD) signals were measured with an echoplanar imaging sequence (echo time: $30 \mathrm{~ms}$, repetition time: $1000 \mathrm{~ms}$, field of view: $22 \times 22 \mathrm{~cm}^{2}$, matrix: $64 \times 64$, flip angle: 60 , slice thickness: 3 $\mathrm{mm}$, gap: $0 \mathrm{~mm}$, number of slices: 14 , slice orientation: axial). The fMRI slices covered the occipital lobe. A high-resolution 3D structural data set (3D MPRAGE; $1 \times 1 \times 1 \mathrm{~mm}^{3}$ resolution) was collected in the same session before the functional scans. All four subjects participated in four fMRI sessions for the retinotopic mapping, the attendto-fixation condition, the attend-to-stimulus condition and the control experiment, respectively.

MRI data processing and analysis. The anatomical volume for each subject in the retinotopic mapping session was transformed into the anterior commissure-posterior commissure space. The cortical surface was extracted and then inflated using BrainVoyager QX. Functional volumes in all the sessions for each subject were preprocessed which included 3D motion correction, linear trend removal, and highpass $(0.015 \mathrm{~Hz})$ (Smith et al., 1999) filtering using BrainVoyager QX. The images were then aligned to the anatomical volume in the retinotopic mapping session and transformed into the AC-PC space. The first $10 \mathrm{~s}$ of BOLD signals were discarded to minimize transient magnetic-saturation effects.

A general linear model procedure was used for selecting ROIs. The ROIs in V1 and V2 were defined as areas that responded more strongly to the flickering ring than the blank interval $\left(p<10^{-4}\right.$, Bonferroni corrected), and were confined by the V1/V2/V3 boundaries defined by the retinotopic mapping experiment. For three subjects, the fMRI slices covered their posterior intraparietal sulcus (pIPS). Part of this area could be activated by the flicking ring $\left(p<10^{-2}\right)$ and served as a ROI outside the retinotopic areas.

The event-related BOLD signals were calculated separately for each ROI in each subject and experiment, following the method used by Larsson et al. (2006) and Liu et al. (2007). For each fMRI scan, the time course of MR signal intensity was first extracted by averaging the data across all the voxels within the predefined ROI and then normalized by the mean intensity across the scan. Event-related averages were then performed for each of the three trial types (same, different and blank) by averaging 12 time points starting at the test stimulus. The

\section{A. Attend to fixation}
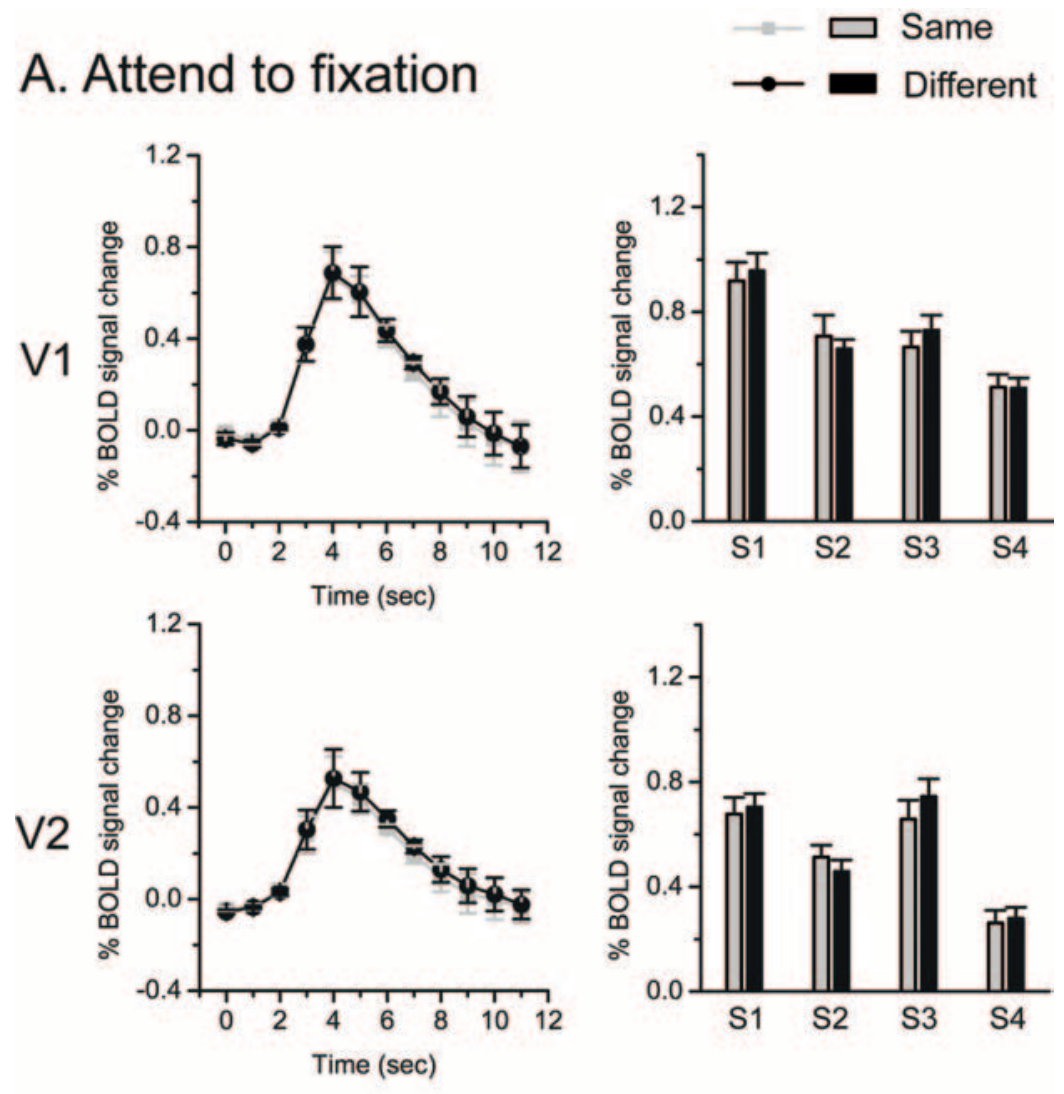

\section{B. Attend to stimulus}
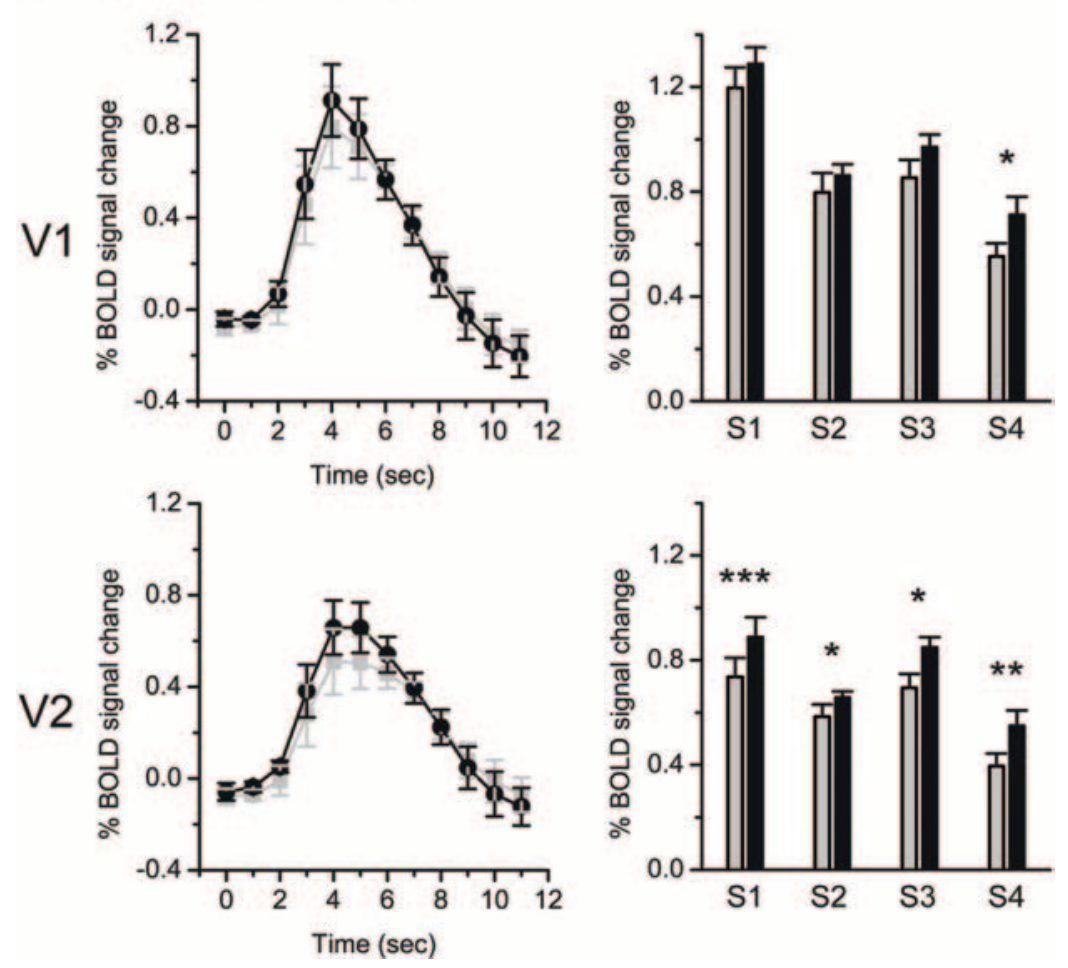

Figure 2. fMRI results in the main experiment. $\boldsymbol{A}$, Attend-to-fixation condition. $\boldsymbol{B}$, Attend-to-stimulus condition. For each experimental condition, left column shows the time courses of BOLD signals evoked by test stimuli that were presented at time point 0 . The signals were averaged across four subjects in V1 and V2. Error bars denote 1 SEM calculated across subjects at each time point. Right column shows fMRI response amplitudes to test stimuli for individual subjects. Asterisks indicate a statistically significant difference between the fMRI response amplitudes to the same and the different test stimuli ( ${ }^{*} p<0.05$; ${ }^{* *} p<0.01$; $\left.{ }^{* * *} p<0.001\right)$. Error bars denote 1 SEM calculated across scans for each subject. 


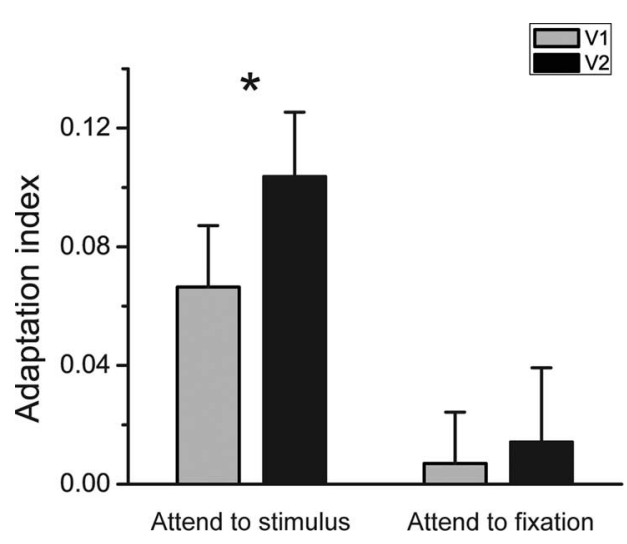

Figure 3. Adaptation indices of V1 and V2 averaged across four subjects in the attend-tofixation condition and the attend-to-stimulus condition. Asterisks indicate a statistically significant difference between the adaptation indices of V1 and V2 ( $\left.{ }^{*} p<0.05\right)$. Error bars denote 1 SEM calculated across subjects.

average response to the blank trials was subtracted from the averages of the same and different trials to isolate the response to the test stimulus.

The peak fMRI response to the test stimulus was used as a measure of the response amplitude. We also computed an adaptation index $I_{\mathrm{A}}$ to quantify how much the measured response changed after adaptation, relative to the overall response to the stimuli in each visual area. The index was calculated as follows: $I_{\mathrm{A}}=\left(A_{\text {different }}-A_{\text {same }}\right) /\left(A_{\text {different }}+A_{\text {same }}\right)$, where $A_{\text {different }}$ is the mean amplitude of the responses to the different test stimulus, and $A_{\text {same }}$ is the mean amplitude of the responses to the same test stimulus. This index could range from -1 to 1 and was positive whenever the mean response to the different test stimulus was greater than the mean response to the same test stimulus. A large adaptation index of a ROI means a strong adaptation effect, with the implication of a large proportion of border ownershipselective neurons in that ROI.

Eye movement recording. Eye movements were recorded at $60 \mathrm{~Hz}$ with an iView X RED eye tracker (SensoMotoric Instruments) in a psychophysics lab when subjects viewed the same stimuli as those in the magnet. Inspection of the eye movement data revealed that all subjects were able to maintain stable fixation throughout the experiment and most of their gaze positions were within $0.5^{\circ}$ radius of the fixation point.

\section{Results}

\section{Behavioral data}

Behavioral data showed that the subjects strictly followed the instructions. In the attend-to-stimulus condition, their responses to the test stimuli were both accurate and fast. The correct rates for both the same and different trials were $>97 \%$. The reaction times (mean \pm SEM) were $519 \pm 31 \mathrm{~ms}$ for the same trials and $517 \pm 33 \mathrm{~ms}$ for the different trials. In the attend-to-fixation condition, for the same trials and the different trials, the correct rates (mean $\pm \mathrm{SEM}$ ) of discriminating the luminance change were $77 \pm 3 \%$ and $75 \pm 3 \%$ respectively, and the reaction times (mean \pm SEM) were $451 \pm 16 \mathrm{~ms}$ and $443 \pm 19 \mathrm{~ms}$, respectively. For all these measurements, there was no significant difference between the two types of trials.

\section{Border ownership adaptation in V1 and V2}

fMRI signals were extracted from the ROIs in V1 and V2 and were selectively averaged according to the trial type. The peak fMRI response to the test stimulus was used as a measure of the response amplitude. We hypothesize that, if a cortical area contains neurons selective to border ownership, according to the fMRI adaptation logic, the area should show a higher response to the test stimulus that is different from the adapting stimulus (differ- ent trial) than to the test stimulus that is the same as the adapting stimulus (same trial).

In the attended-to-fixation condition (Fig. $2 A$ ), none of the four subjects showed a significant adaptation effect, a higher response in the different trial than in the same trial, in both V1 and V2. In the attend-to-stimulus condition (Fig. $2 B$ ), the adaptation effect in V1 was weak and unreliable. Only one subject (S4) showed a significant adaptation effect $(t=3.165, p=0.025)$. However, the adaptation effect in V2 was strong and consistent across all the four subjects (S1: $t=7.212, p<0.001 ; \mathrm{S} 2: t=2.679$, $p=0.044$; S3: $t=2.891, p=0.034$; S4: $t=5.095, p=0.004)$. These results demonstrate that $\mathrm{V} 2$ is a critical area for the processing of border ownership and this processing is largely dependent on attention.

The adaptation index can be used for comparing border ownership selectivity between different cortical areas. A large index of a cortical area means a strong adaptation effect and suggests a large proportion of border ownership-selective neurons in this area (Larsson et al., 2006; Ashida et al., 2007; Fang et al., 2007). In the attend-to-stimulus condition, the adaptation index of $\mathrm{V} 2$ was significantly larger than that of V1 $(t=4.958, p=0.016)$. The adaptation index of V1 was significantly larger than zero $(t=$ $3.214, p=0.049$ ) although the V1 adaptation effect was not consistently exhibited at the individual subjects level. But in the attend-to-fixation conditions, the adaptation indices of both V1 and V2 were not significantly larger than zero (no adaptation effect), and there was no significant difference between V1 and V2 (Fig. 3). These results further suggest that V2 plays a more important role than $\mathrm{V} 1$ in border ownership processing.

\section{Control experiment}

It could be argued that the adaptation effect we found in V2 was due to the image difference (the contextual information itself) between the adapting stimulus and the test stimulus, rather than the perceived border ownership difference. In the main experiment, the separation between the ROI ring and the image regions that provide the contextual information was at least $2.5^{\circ}$ (Fig. $1 C$ ), which is much larger than the classical receptive field sizes $\left(0.5^{\circ}\right.$ and $\left.1.5^{\circ}\right)$ of $\mathrm{V} 1$ and $\mathrm{V} 2$ neurons at the eccentricity of the ring (Smith et al., 2001). The human population receptive field size estimates (Dumoulin and Wandell, 2008) also agree well with the electrophysiological measurements. All the evidence suggests that the V1 and V2 neurons in the ROIs cannot respond directly to the distant contextual information and were presumably driven by locally identical stimuli.

However, Cornelissen et al. (2006) argued that, within V1 and $\mathrm{V} 2$, the fMRI signal from one subregion could be enhanced by a visual stimulus that stimulated another remote subregion. Thus, the observed effect in the ROI in V2 could be an artifactual consequence of the contextual change, but not the border ownership adaptation. We performed the control experiment to rule out this potential confound. The stimuli used in the control experiment were modified from the stimuli in the main experiment. Only the image region providing the contextual information and an annular interior part of the radial grating having the same size as the ROI stimulus were kept. Thus, the image difference between the stimulus pair in the control experiment was the same as that in the main experiment. But the borders in the annular interior part of the radial grating did not have a clear ownership. The experimental procedure was the same as the attend-to-stimulus condition in the main experiment. We did not find any adaptation effect in either V1 or V2: there was no significant difference between same trials and different trials (Fig. 4). 


\section{Discussion}

It is well known that spatial attention can strongly modulate fMRI signals in the visual cortex, as early as V1 (Tootell et al., 1998; Brefczynski and DeYoe, 1999). The observed fMRI signal difference between same trials and different trials in the main experiment could have reflected an attentional signal elicited by the change in the stimulus, rather than selective adaptation to border ownership. Although we found that border ownership adaptation was dependent on attention, we have several reasons to argue against that it is a pure attentional effect. First, there was no significant difference in the behavioral data (reaction time and correct rate) between same trials and different trials. More attention allocated to the test stimulus in the different trials could have presumably resulted in a better performance (Posner, 1980). Second, the image difference in the stimulus pair in the control experiment was the same as that in the main experiment. If the fMRI signal difference in the main experiment reflected an attentional signal elicited by the physical change of the stimulus, we should have observed a similar signal difference in the control experiment. But our data show that this is not the case. Third, for three subjects, fMRI slices covered their pIPS, one of the core regions of the dorsal attention network (Kastner and Ungerleider, 2000; Corbetta and Shulman, 2002), which is the putative source of attentional signals to visual cortex (Moore and Armstrong, 2003). Although the pIPS showed a robust response to the presentation of the same and different test stimuli, there was no difference between them, which suggested that no more attention was paid to or elicited by the different test stimulus than the same test stimulus.

Previous human fMRI studies found that some higher-level visual areas, the LOC and FFA, were sensitive to a change of border ownership (Kourtzi and Kanwisher, 2001; Andrews et al., 2002). Baylis and Driver (2001) showed that the inferotermporal (IT) cortex of awake, behaving monkeys contains neurons that were selective to border ownership. Since monkey IT and human LOC and FFA are the brain areas critically involved in object and face recognition, the border ownership selectivity in these areas might be an epiphenomenon of the neural processes underlying object recognition. It is possible that earlier visual areas resolve border ownership and provide input to higher visual areas (Rubin, 2001). Zhou et al. (2000) reported that $>50 \%$ of the neurons in monkey V2 showed a border ownership selectivity, which provides some tentative support for the early computation idea. In a human psychophysical study, von der Heydt et al. (2005) showed a border-ownership-dependent tilt aftereffect pointing to the existence of border-ownership selective neurons at early stages in the human visual cortex. Consistent with these studies, our fMRI study demonstrated the important role of V2 in mediating the processing of border ownership.

We found that attention is critical for the neural selectivity of border ownership: when subjects did a very demanding fixation task, the border ownership adaptation effect was nearly abolished in their early visual cortex. Qiu et al. (2007) reported that, although the neuronal responses to border ownership in V2 were strongly modulated by attention, there were still some neurons whose selectivity to border ownership was independent of attention. This discrepancy could be attributed to many factors. In addition to the species difference (human vs monkey) and stimulus difference, one important factor is the attentional task difference. Subjects did the fixation task throughout an $\mathrm{fMRI}$ run $(\sim 7 \mathrm{~min})$ and they reported having little awareness of the border ownership assignment. It might be more proper to claim that border ownership assignment depends on visual awareness. In Qiu et al. (2007), the onset of a test stimulus could have recruited some bottom-up (exogenous) attention, although the stimulus was cued to be ignored. Another important factor is the measure difference (fMRI adaptation vs single-unit recording). Our claim that border ownership selectivity depends on attention is based on the finding of attention-dependent border ownership selective adaptation. It might be argued that border ownership signals do not depend on attention, but their adaptation does. Although we cannot completely rule out this possibility and it is true that some kind of adaptation requires attention (Yeh et al., 1996), it is widely accepted that selective adaptation originates from neuronal selectivity and many studies have demonstrated that they can be modulated similarly by attention (Murray and Wojciulik, 2004; Clifford and Rhodes, 2005).

The attentional effect indicates that border ownership processing depends on the modulation from higher-level cortical areas. This modulation could be realized in two different ways. 
One way is that attention enhances V2 activity, and V2 by itself generates the border ownership signal through intracortical interactions, as suggested in a computational model by Zhaoping (2005). The other way is that, higher visual areas whose activity is largely dependent on attention, integrate contextual information, determine the border ownership and feed back to V2.

In visual information processing, border ownership assignment is closely related to figure-ground organization. Electrophysiological studies (Zipser et al., 1996; Lee et al., 1998) (but see Rossi et al., 2001) have found enhancement of texture-evoked activity in figure regions compared with the ground region in V1 neurons. In our study, the border ownership changes were accompanied by a change of figure-ground organization. However, the weak and unreliable adaptation effect in $\mathrm{V} 1$ indicates that there was no robust sensitivity to the figure-ground change. Zipser et al. (1996) and Lee et al. (1998) used line-textured figures that were centered on the receptive field. Thus, the enhancement in figure regions reflected the activity of neurons that are selective for the texture orientation, not the figure boundary. However, the figures and ground in our stimuli were objects of uniform color. Because it is known that most cortical neurons respond very little to uniform stimuli (Hubel and Wiesel, 1968), figure-ground information in our study is likely coded only in the border responses. It remains to be examined whether the figure-ground effect can be revealed in V1 by using line-textured stimuli and $\mathrm{AMRI}$ adaptation technique.

Consistent with previous studies (Albright and Stoner, 2002), our results suggest that neurons in early visual cortex integrate the image context far beyond the classical receptive field, and add weight to the claim that high-level visual computations and representations involve neural activity in early visual cortex (Lee et al., 1998). Although it has been shown that fMRI signals in human early visual cortex can be modulated by contextual information (ZengerLandolt and Heeger, 2003; Sasaki and Watanabe, 2004; Murray et al., 2006; Boyaci et al., 2007), we demonstrate a context-dependent fMRI adaptation effect that provides clear evidence of border ownership selectivity at a subvoxel level in human early visual cortex. Together with other evidence, the resolution of border ownership involves computations in early as well as high-level cortical areas. In future research, asking how these computations are done by inter- and intracortical cooperation will be of great importance to understand how human visual system parses images and recognizes objects.

\section{References}

Albright TD, Stoner GR (2002) Contextual influences on visual processing. Annu Rev Neurosci 25:339-379.

Andrews TJ, Schluppeck D, Homfray D, Matthews P, Blakemore C (2002) Activity in the fusiform gyrus predicts conscious perception of Rubin's vase-face illusion. Neuroimage 17:890-901.

Ashida H, Lingnau A, Wall MB, Smith AT (2007) fMRI adaptation reveals separate mechanisms for first-order and second-order motion. J Neurophysiol 97:1319-1325.

Baylis GC, Driver J (2001) Shape-coding in IT cells generalizes over contrast and mirror reversal, but not figure-ground reversal. Nat Neurosci 4:937-942.

Boyaci H, Fang F, Murray SO, Kersten DJ (2007) Responses to lightness variations in early human visual cortex. Curr Biol 17:989-993.

Brefczynski JA, DeYoe EA (1999) A physiological correlate of the "spotlight" of visual attention. Nat Neurosci 2:370-374.

Buracas GT, Boynton GM (2002) Efficient design of event-related fMRI experiments using $\mathrm{M}$-sequences. Neuroimage 16:801-813.

Clifford CWG, Rhodes G (2005) Fitting the mind to the world: adaptation and after-effects in high-level vision. New York: Oxford UP.

Corbetta M, Shulman GL (2002) Control of goal-directed and stimulusdriven attention in the brain. Nat Rev Neurosci 3:201-215.

Cornelissen FW, Wade AR, Vladusich T, Dougherty RF, Wandell BA (2006) No functional magnetic resonance imaging evidence for brightness and color filling-in in early human visual cortex. J Neurosci 26:3634-3641.
Driver J, Baylis GC (1996) Edge-assignment and figure-ground segmentation in short-term visual matching. Cognit Psychol 31:248-306.

Dumoulin SO, Wandell BA (2008) Population receptive field estimates in human visual cortex. Neuroimage 39:647-660.

Engel SA, Glover GH, Wandell BA (1997) Retinotopic organization in human visual cortex and the spatial precision of functional MRI. Cereb Cortex 7:181-192.

Fang F, Murray SO, Kersten D, He S (2005) Orientation-tuned fMRI adaptation in human visual cortex. J Neurophysiol 94:4188-4195.

Fang F, Murray SO, He S (2007) Duration-dependent fMRI adaptation and distributed viewer-centered face representation in human visual cortex. Cereb Cortex 17:1402-1411.

Hubel DH, Wiesel TN (1968) Receptive fields and functional architecture of monkey striate cortex. J Physiol 195:215-243.

Kastner S, Ungerleider LG (2000) Mechanisms of visual attention in the human cortex. Annu Rev Neurosci 23:315-341.

Kourtzi Z, Kanwisher N (2001) Representation of perceived object shape by the human lateral occipital complex. Science 293:1506-1509.

Krekelberg B, Boynton GM, van Wezel RJA (2006) Adaptation: from single cells to BOLD signals. Trends Neurosci 29:250-256.

Larsson J, Landy MS, Heeger DJ (2006) Orientation-selective adaptation to first- and second-order patterns in human visual cortex. J Neurophysiol 95:862-881.

Lee TS, Mumford D, Romero R, LammeVAF (1998) The role of the primary visual cortex in higher level vision. Vision Res 38:2429-2454.

Liu T, Larsson J, Carrasco M (2007) Feature-based attention modulates orientation-selective responses in human visual cortex. Neuron 55:313-323.

Moore T, Armstrong KM (2003) Selective gating of visual signals by microstimulation of frontal cortex. Nature 421:370-373.

Murray SO, Wojciulik E (2004) Attention increases neural selectivity in the human lateral occipital complex. Nat Neurosci 7:70-74.

Murray SO, Boyaci H, Kersten D (2006) The representation of perceived angular size in human primary visual cortex. Nat Neurosci 9:429-434.

Nakayama K, Shimojo S, Silverman GH (1989) Stereoscopic depth: its relation to image segmentation, grouping, and the recognition of occluded objects. Perception 18:55-68.

Posner MI (1980) Orienting of attention. Q J Exp Psychol 32:3-25.

Qiu FT, von der Heydt R (2005) Figure and ground in the visual cortex: V2 combines stereoscopic cues with Gestalt rules. Neuron 47:155-166.

Qiu FT, Sugihara T, von der Heydt R (2007) Figure-ground mechanisms provide structure for selective attention. Nat Neurosci 10:1492-1499.

Rossi AF, Desimone R, Ungerleider LG (2001) Contextual modulation in primary visual cortex of macaques. J Neurosci 21:1698-1709.

Rubin N (2001) Figure and ground in the brain. Nat Neurosci 4:857-858.

Sasaki Y, Watanabe T (2004) The primary visual cortex fills in color. Proc Natl Acad Sci U S A 101:18251-18256.

Sereno MI, Dale AM, Reppas JB, Kwong KK, Belliveau JW, Brady TJ, Rosen BR, Tootell RBH (1995) Borders of multiple visual areas in humans revealed by functional magnetic resonance imaging. Science 268:889-893.

Smith AM, Lewis BK, Ruttimann UE, Ye FQ, Sinnwell TM, Yang Y, Duyn JH, Frank JA (1999) Investigation of low frequency drift in fMRI signal. Neuroimage 9:526-533.

Smith AT, Singh KD, Williams AL, Greenlee MW (2001) Estimating receptive field size from fMRI data in human striate and extrastriate visual cortex. Cereb Cortex 11:1182-1190.

Tootell RB, Hadjikhani N, Hall EK, Marrett S, Vanduffel W, Vaughan JT, Dale AM (1998) The retinotopy of visual spatial attention. Neuron 21:1409-1422.

von der Heydt R, Macuda T, Qiu FT (2005) Border-ownership-dependent tilt aftereffect. J Opt Soc Am A 22:2222-2229.

Yeh SL, Chen IP, De Valois KK, De Valois RL (1996) Figural aftereffects and spatial attention. J Exp Psychol Hum Percept Perform 22:446-460.

Zenger-Landolt B, Heeger DJ (2003) Response suppression in V1 agrees with psychophysics of surround masking. J Neurosci 23:6884-6893.

Zhaoping L (2005) Border ownership from intracortical interactions in visual area V2. Neuron 47:143-153.

Zhou H, Friedman HS, von der Heydt R (2000) Coding of border ownership in monkey visual cortex. J Neurosci 20:6594-6611.

Zipser K, Lamme VA, Schiller PH (1996) Contextual modulation in primary visual cortex. J Neurosci 16:7376-7389. 